\title{
Rapid Simultaneous Enhancement of Visual Sensitivity and Perceived Contrast during Saccade Preparation
}

\author{
Martin Rolfs ${ }^{1,3}$ and Marisa Carrasco ${ }^{1,2}$ \\ ${ }^{1}$ Department of Psychology and ${ }^{2}$ Center for Neural Science, New York University, New York, New York 10003, and ${ }^{3}$ Laboratoire de Psychologie Cognitive, \\ Aix-Marseille Université-CNRS, 13331 Marseille, France
}

Humans and other animals with foveate vision make saccadic eye movements to prioritize the visual analysis of behaviorally relevant information. Even before movement onset, visual processing is selectively enhanced at the target of a saccade, presumably gated by brain areas controlling eye movements. Here we assess concurrent changes in visual performance and perceived contrast before saccades, and show that saccade preparation enhances perception rapidly, altering early visual processing in a manner akin to increasing the physical contrast of the visual input. Observers compared orientation and contrast of a test stimulus, appearing briefly before a saccade, to a standard stimulus, presented previously during a fixation period. We found simultaneous progressive enhancement in both orientation discrimination performance and perceived contrast as time approached saccade onset. These effects were robust as early as 60 ms after the eye movement was cued, much faster than the voluntary deployment of covert attention (without eye movements), which takes $\sim 300$ ms. Our results link the dynamics of saccade preparation, visual performance, and subjective experience and show that upcoming eye movements alter visual processing by increasing the signal strength.

\section{Introduction}

Rapid eye movements, called saccades, largely shape the way we see. Like a flick of the wrist swivels a hand-held camera, saccades swiftly point the fovea to locations of interest, ensuring high-acuity vision for the scene's relevant information. The enhanced processing of the targets of saccades starts even before the eyes begin to move (Kowler et al., 1995; Deubel and Schneider, 1996)-observers show pronounced improvements in performance in visual discrimination tasks, starting $\sim 100$ ms before a saccade (Castet et al., 2006; Montagnini and Castet, 2007; Deubel, 2008; Rolfs et al., 2011).

A series of microstimulation and inactivation studies in monkeys has provided clues as to how these presaccadic perceptual benefits arise. Neural activity in saccade-related brain areas rapidly gates processing in visual cortex (Armstrong and Moore, 2007) and augments visual performance at the corresponding retinal locations (Moore and Fallah, 2001; Cavanaugh and Wurtz, 2004; Wardak et al., 2004, 2006; Müller et al., 2005; Balan and Gottlieb, 2009; Lovejoy and Krauzlis, 2010). Consequently, it has been proposed that signals emerging from saccade-related brain areas provide feedback to visual cortex to change the gain of

\footnotetext{
Received May 30, 2012; revised July 13, 2012; accepted Aug. 4, 2012.

Author contributions: M.R. and M.C. designed research; M.R. performed research; M.R. and M.C. contributed unpublished reagents/analytic tools; M.R. analyzed data; M.R. and M.C. wrote the paper.

M.R. is supported by the 7th Framework Program of the European Commission (Marie Curie International Outgoing fellowship, project 235625); M.C. is supported by NIH Grant R01 EY016200. We thank Alexander L. White for valuable discussion of the data, Eric Castet, Anna Montagnini, Miriam Spering, and members of the Carrasco lab for comments on the manuscript, and Hakan Karsilar and Sarah Lucy Charlesworth Poe for help with data acquisition. The authors declare no competing financial interests.

Correspondence should be addressed to Dr. Martin Rolfs, Department of Psychology, New York University, 6 Washington Place, New York, NY 10003. E-mail: martin.rolfs@nyu.edu.

DOI:10.1523/JNEUROSCI.2676-12.2012

Copyright $\odot 2012$ the authors $\quad 0270-6474 / 12 / 3213744-09 \$ 15.00 / 0$
}

responses to visual input (Hamker, 2005), increasing the signal strength as if stimulus saliency itself were increased. Many areas of the monkey brain exhibit increases in the gain of visual responses with saccade preparation (Boch and Fischer, 1983; Chelazzi et al., 1993; Moore et al., 1998; Sheinberg and Logothetis, 2001; Mazer and Gallant, 2003; Li and Basso, 2008), indeed mimicking a change in signal strength, e.g., stimulus contrast. These extraretinal influences on visual processing may alter subjective perception and, if so, reveal similar mechanisms in human observers.

The evidence that covert attention (i.e., without eye movements) alters subjective experience (Carrasco et al., 2004) has completed a triangle of converging evidence from psychophysical findings, electrophysiology, and neuroimaging, which argues that attention enhances the processing of attended sensory information and modifies its very appearance (Treue, 2004). Similarly, evidence that movement preparation alters subjective experience would imply that it modifies sensory processing to create a perceptual experience that integrates visual signals and behavioral priority. Here we reveal this missing link by showing that saccade preparation increases the perceived signal strength-perceived contrast-consistent with enhanced neural representations of visual information at the saccade target.

We implemented a novel behavioral method, inspired by a psychophysical paradigm developed to quantify subjective, phenomenological correlates of covert attention (Carrasco et al., 2004; Liu et al., 2009) objectively and rigorously (Luck, 2004; Treue, 2004; Carrasco, 2009). In this study, to assess effects of saccade preparation, we asked observers to simultaneously judge both the orientation and the contrast of a test stimulus presented at the target of a cued saccadic eye movement relative to a standard stimulus shown during prior fixation (Fig. 1). Given that 


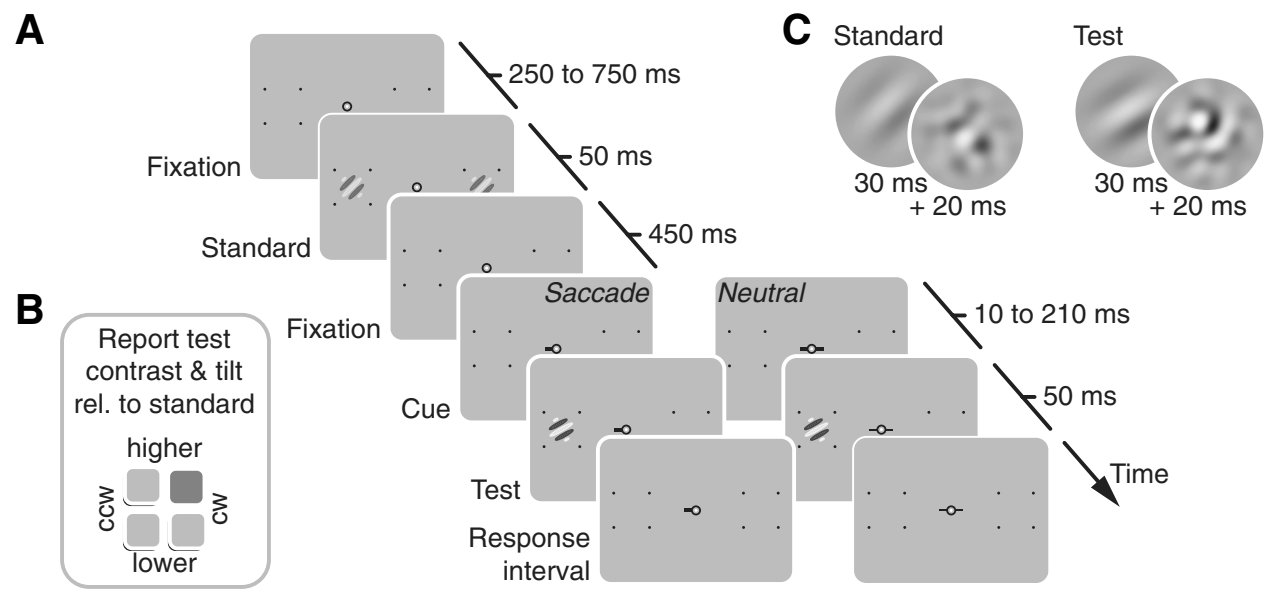

Figure 1. Design of Experiment 1.A, Sequence of events in each trial. Standard stimuli briefly appeared $10^{\circ}$ to the left and to the right of a central fixation spot, followed by a cue and, with a variable delay, a test stimulus was shown on one side only. In the saccade condition, the cue pointed toward the test location and observers made a saccade as quickly as possible. In the neutral condition, the cue was uninformative with respect to the test location and observers held fixation. $\boldsymbol{B}$, Standard and test stimuli consisted of a Gabor patch and a subsequent orientation-noise mask. Standard stimuli had an orientation of $\pm 45^{\circ}$ and a contrast of $22.4 \%$. Test stimulus orientation differed slightly from the standard, in clockwise (CW) or counterclockwise (CCW) direction, and we varied their contrast around the standard contrast. $\boldsymbol{C}$, After the stimulus sequence, once an appropriate eye movement was made, observers reported the orientation and contrast of the test stimulus relative to the standard with a single button press (here, higher contrast and clockwise, highlighted in dark gray) using the hand corresponding to the side of the test stimulus.

performance in orientation discrimination tasks is mediated by contrast sensitivity (Carrasco et al., 2000; Pestilli et al., 2009; Herrmann et al., 2010), we simultaneously assessed the effects of saccade preparation on both contrast sensitivity and perceived contrast.

\section{Materials and Methods}

Participants. We tested four observers (age 25-33 years, male, right-eye dominant; the first author and two psychophysically trained and one untrained observer; one left-handed) in Experiment 1, and three of them also participated in Experiment 2. We tested four new observers (age 21-23 years, female, right-eye dominant, psychophysically untrained; one left-handed) in Experiment 3. All observers but the author were naive regarding the purpose of the study, had normal or corrected-tonormal vision, and signed informed consent forms before participation. The NYU Institutional Review Board approved the study and experiments were performed in agreement with the Helsinki declaration.

Setup. Observers sat in a silent and dimly lit room, head positioned on a chin rest. We presented stimuli at $57 \mathrm{~cm}$ distance on a gammalinearized 22-inch Sony GDM-F520 screen $(1280 \times 960$ pixels, 100 $\mathrm{Hz}$ vertical refresh). An EyeLink 1000 Desktop Mount (SR Research) recorded the right eye's gaze position. A computer running MATLAB (MathWorks) with standard toolboxes (Brainard, 1997; Pelli, 1997; Cornelissen et al., 2002) controlled stimulus presentation and response collection.

Experiment 1: presaccadic changes in sensitivity and appearance. Before each trial, a fixation stimulus (red dot with diameter of $0.2^{\circ}$ in a black annulus with diameter: $0.7^{\circ}$ ) appeared at the center of a uniform gray display. When participants kept fixation within a $1.5^{\circ}$ radius for $200 \mathrm{~ms}$, the dot turned white and the trial started with a presentation of two placeholders indicating the locations of the upcoming stimuli (Fig. $1 \mathrm{~A}$ ). Placeholders were four black dots (diameter: $0.2^{\circ}$ ) forming the corners of a square with $5^{\circ}$ side length, centered on the future stimulus locations, $10^{\circ}$ left and right of fixation. After an interval of $250-750 \mathrm{~ms}$, randomly drawn from a uniform distribution, we flashed two identical standard stimuli simultaneously at the two stimulus locations. Five hundred milliseconds after the onset of the standard stimulus, a line pointed $0.5^{\circ}$ away from fixation, cueing a saccade to the upcoming target location (randomly left or right). At a variable time after the cue (10-210 ms in steps of $50 \mathrm{~ms}$; in $63.9 \%$ before the saccade), we flashed a single test stimulus at the saccade target location. Once the eye landed in a circular target area (enveloping the landmarks, i.e., $3.53^{\circ}$ radius), a tone initiated the response interval. In a two-by-two alternative forced-choice task, observers reported the test stimulus' relative orientation (clockwise or counterclockwise of the standard) and contrast (higher or lower than the standard) with a single button press, using one of four keys (Fig. 1B).

Observers also participated in a neutral condition, in which the cue pointed in both directions and observers had to maintain fixation throughout the trial until the response tone sounded. To have stimulusresponse compatible conditions, observers gave responses for test stimuli on the left side of the screen with the left hand (keys A, S, Z, and X on the keyboard) and test stimuli on the right side with the right hand (keys 5, 6, 2 , and 3 on the number pad). We monitored eye movements throughout the trial - when fixation was lost in the fixation period or the eyes did not land in the target area within $400 \mathrm{~ms}$ after the saccade cue, the trial was aborted and repeated in random order at the end of a block (5.7\% of all trials).

Both standard and test stimuli consisted of $30 \mathrm{~ms}$ Gabor patches and a subsequent $20 \mathrm{~ms}$ orientation-noise mask (Fig. 1C), increasing the difficulty of the orientation judgments. Standard stimulus Gabors had 22.4\% contrast, a $\pm 45^{\circ}$ orientation, and a Gaussian envelope with $0.5^{\circ} \mathrm{SD}$. To avoid aftereffects, we randomized their phase (range of $2 \pi$ ) and spatial frequency ( 1 or $1.5 \mathrm{cpd}$ ). The test stimulus Gabor was identical to the standard stimulus Gabors on any given trial, except for its orientation (rotated clockwise or counterclockwise of the standard) and contrast (range of 0.9 log units in seven equidistant steps around standard contrast). Orientation-noise masks were patches of Gaussian pixel noise (SD corresponding to the contrast of the preceding stimulus), bandpassfiltered from half to twice the spatial frequency of the stimulus, and enveloped in the same Gaussian window.

After a practice session training procedural aspects of the task, each observer completed 9800 trials in $141 \mathrm{~h}$ sessions (10 blocks of 70 trials each). Saccade trials and neutral trials were blocked (flights of five or 10 blocks in a row) to avoid prolonged saccade latencies. The order of conditions was counter-balanced within and randomized across observers. Before the first session and several times during the study, we conducted a short pretest ( 1 block of 70 trials) to obtain the $82 \%$ orientation discrimination threshold for test stimuli presented before the saccade, using a single QUEST (Watson and Pelli, 1983) staircase (in the initial sessions of three participants, we also adjusted the set of test contrasts, but we abandoned this strategy as a range centered on the standard contrast covered the dynamic range of responses well). The orientation difference between test and standard stimulus was $11.7 \pm 0.8^{\circ}$. The procedure in the pretest was identical to the saccade task in the main experiment, except that we provided auditory feedback on performance in the orientationdiscrimination task. 
Experiment 2: spatial cueing, but no saccade. In this experiment, all visual parameters and the task were the same as in Experiment 1 (Fig. $1 A$ ), except that the neutral condition was now paired with a covert attention condition. The covert attention condition was visually identical to the saccade condition in Experiment 1, but observers attended covertly to the indicated target location, without moving the eyes. In both cases, the cue was $100 \%$ valid. We obtained the $82 \%$ orientation discrimination threshold for test stimuli using a single QUEST staircase in the same way as in Experiment 1. The orientation difference between test and standard stimulus was $9.7 \pm 1.1^{\circ}$. Three observers ( $\mathrm{MR}, \mathrm{AW}, \mathrm{AB}$ ) who also participated in Experiment 1 completed a total of 5600 trials in eight $1 \mathrm{~h}$ sessions each, half in the covert and half in the neutral condition, blocked in a counterbalanced order. (Observer HK left the U.S.A. and was thus unavailable for further testing.) Trial repetitions after unsolicited eye movements occurred in $2.6 \%$ of all trials.

Experiment 3: no masks and no performance task. The only differences from Experiment 1 were the following: Instead of composing standard and test stimuli of a Gabor and a mask, stimuli consisted only of a $50 \mathrm{~ms}$ Gabor. Observers reported whether the test stimulus was higher or lower in contrast than the standard for test stimuli on the left side with the left hand (keys $\mathrm{S}$ and $\mathrm{X}$ ) and for test stimuli on the right side with the right hand (number keys 5 and 2 on number pad). The test stimulus' orientation was irrelevant to the observer, but randomly $\pm 10^{\circ}$ off the standard's orientation to mimic the visual stimulation in Experiment 1. In eight $1 \mathrm{~h}$ sessions, each observer completed a total of 5600 trials, half in the saccade and half in the neutral condition; the order of blocks was counterbalanced. Because of incorrect eye movements, $7.7 \%$ of all trials were repeated.

Data preprocessing. Using low-pass filtered eye position data, we detected saccades offline based on their $2 \mathrm{D}$ velocity. We computed smoothed eye velocities using a moving average over five subsequent 1 ms eye position samples in a trial (Engbert and Mergenthaler, 2006). Saccades exceeded the median velocity by 5 SDs for at least $8 \mathrm{~ms}$. Overshoots in saccades often result in the detection of two saccades; thus, we merged events separated by $10 \mathrm{~ms}$ or less into a single saccade. Response saccades were the first saccade leaving a fixation region and landing inside a target area (radii of $2.5^{\circ}$ ). We rejected trials in which observers used the wrong hand for the report, trials that contained blinks, no response saccades starting $70-400 \mathrm{~ms}$ after saccade cue onset, and saccades larger than $1.0^{\circ}$ before a response saccade or within $200 \mathrm{~ms}$ after the presentation of the test stimulus in the fixation conditions. Based on these criteria, data analyses included a total of 36,040 trials (or 91.9\%) in Experiment 1 and a total of 19,599 trials (or 87.5\%) in Experiment 3. In Experiment 2, data analyses included a total of 16,158 trials (or 96.2\%).

Data analysis. We determined observers' sensitivity in the orientationdiscrimination task: $d^{\prime}=z$ (hit rate) $-z$ (false alarm rate). A clockwise response to a clockwise stimulus (arbitrarily) defined a hit and a clockwise response to a counterclockwise stimulus defined a false alarm. To determine points of subjective equality (PSEs), we fitted cumulative Gaussians functions with four parameters [mean $(\mu)$, standard deviation $(\delta)$, and lower and upper asymptote ( $\gamma$ and $\lambda$, respectively)] to each observer's contrast reports, using maximum-likelihood estimation with no prior assumptions about parameter values (Wichmann and Hill, 2001a). The PSE is the contrast level at which the fit estimates both response alternatives (test higher or lower in contrast) to be equally likely, i.e., the test stimulus subjectively appeared to have the same contrast as the standard stimulus.

In the analysis of perceptual reports, we derived neutral baselines for each time window before a saccade, ensuring identical test stimulus parameters as in the saccade condition. We did this in three steps, and separately for each observer and each saccade-locked time window. First, for each combination of cue-test interval $i$ and test contrast $c$, we determined the number of trials available in both the saccade and the neutral condition, resulting in matrices of numbers of trials $\mathbf{S}_{i, c}$ and $\mathbf{N}_{i, c}$, respectively, as well as a matrix $\mathbf{R}_{i, c}$, containing the number of a certain report in the neutral condition (correct or higher, for performance and perceived contrast, respectively). Next, we divided $S_{i, c}$ by its maximum value to obtain a matrix of weights, $\mathbf{W}_{i, c}$. Finally, we multiplied $\mathbf{W}_{i, c}$ element-wise by both $\mathbf{N}_{i, c}$ and $\mathbf{R}_{i, c}$ and rounded all elements to the nearest integer, resulting in matrices $\mathbf{N}_{i, c}^{\prime}$ and $\mathbf{R}_{i, c}^{\prime}$, respectively. These matrices represent the neutral condition such that, for each saccade-locked temporal bin, the prevalence of test stimuli with different combinations of cue-test intervals and test contrasts is identical to that in the saccade condition. $\mathbf{N}_{i, c}^{\prime}$ and $\mathbf{R}_{i, c}^{\prime}$ were then used to compute the neutral baseline values of $d^{\prime}$ and PSE. Note that these control data (plotted in Figs. 3-6) do not themselves evolve across time relative to a (simulated) saccade and there is no point in comparing, say, neutral data $100 \mathrm{~ms}$ before the saccade to neutral data $25 \mathrm{~ms}$ before the saccade. Instead, they are baselines derived to match each temporal bin of the saccade condition in terms of physical stimulus parameters. The relevant comparison is the difference between two conditions (e.g., saccade vs neutral) and how that difference evolves across time.

We bootstrapped each observer's perceptual report data 1000 times by resampling from the binomial distribution with the given number of trials and probability (hit/false alarm rates or proportion of higher contrast reports) as parameters (Wichmann and Hill, 2001b). We then computed the variable of interest ( $d^{\prime}$ or PSE) for each of these bootstrap runs and derived SEMs and CIs from the distribution of means across observers or the differences between these means.

\section{Results}

\section{Saccade preparation enhances performance and perceived contrast}

In the first experiment, we simultaneously assessed the temporal evolution of visual performance in an orientation discrimination task as well as perceived contrast before saccades. To this end, observers compared orientation and contrast of a test stimulus, presented at a saccade target around the time of movement onset, to a standard stimulus, presented beforehand, in a period of fixation (Fig. 1A).

The average saccade latency (measured from cue onset) was $191 \pm 4 \mathrm{~ms}$ (individual means, SDs, and density functions in Fig. $2 A$ ), the average landing site was $9.5 \pm 0.2^{\circ}$ away from the initial fixation spot; thus, saccades slightly undershot the target at $10^{\circ}$ eccentricity. Test stimuli with high contrast or occurring early after the cue reduced both saccade latencies and undershoots (Fig. 2C,D). For the analysis of perceptual reports as a function of time before the saccade, we determined the time between the offset (disappearance) of the test stimulus and the saccade onset for each trial, avoiding any overlap of presaccadic test stimuli with the eye movement. Figure $2 B$ shows a density plot of these test times, stacked for the four participants of this experiment and divided into six presaccadic and three intrasaccadic time windows (color coded from dark gray, long before, to light blue, by the end of the saccade), which ensured sufficient data for each observer in each time window while providing us with high temporal resolution for assessing the changes of performance and perceived contrast before and during saccades.

Because the test stimulus parameters affected saccade latency (Fig. 2C), the distribution of test contrasts and cue-test intervals varied somewhat across the different time windows, creating a potential confound in the time course analysis of orientation discrimination performance and perceived contrast. If, for instance, high-contrast test stimuli occurring some time after the cue resulted in better performance, that could be misinterpreted as a presaccadic perceptual enhancement, as these test stimuli often appeared relatively shortly before the saccade (Fig. 2E). To ensure that our results are due to the impending saccade, and not due to stimulus parameters, we derived a baseline from the data collected in a neutral condition (Fig. 1A), which was interleaved (across blocks) with the saccade condition. In the neutral trials, observers did not make a saccade, but we used the exact same stimulus timing and test stimulus parameters, ensuring that both conditions were run under the exact same degree of temporal 


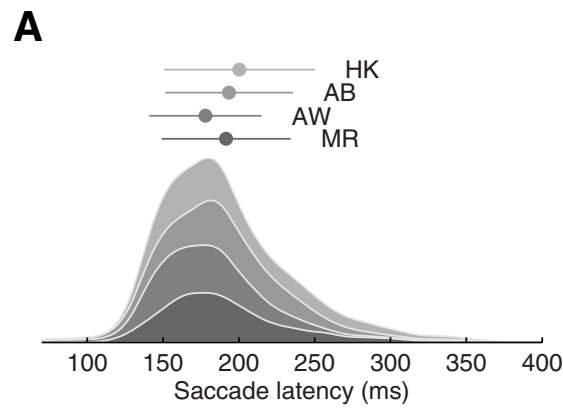

B
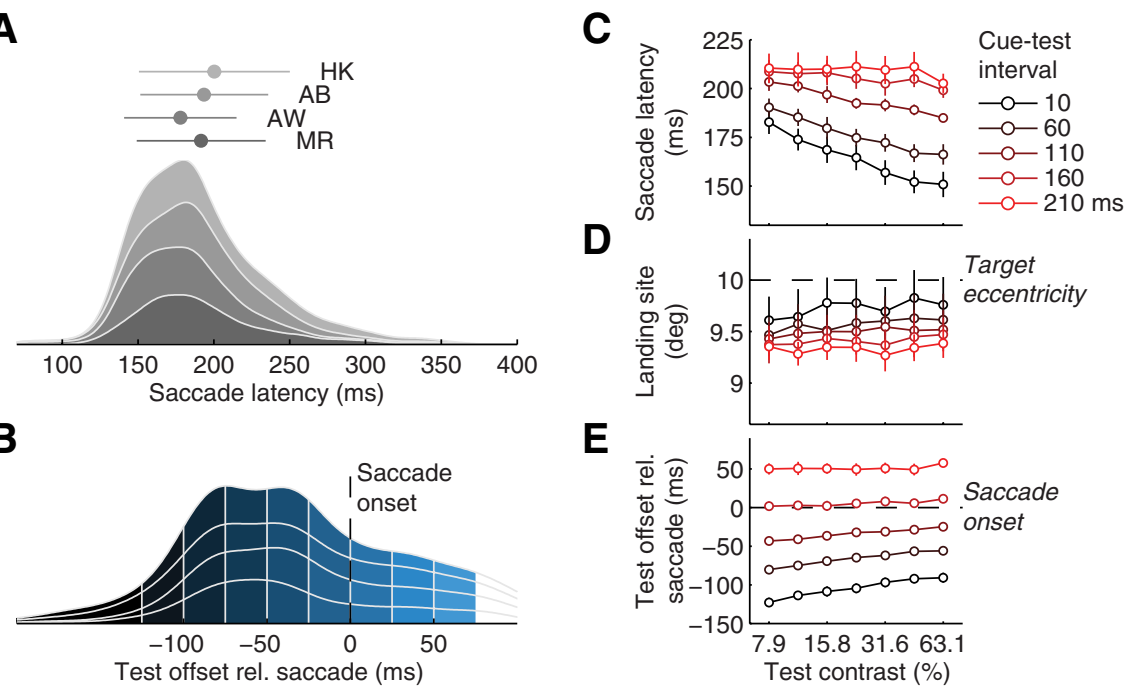

Figure 2. Saccade-related parameters in Experiment 1. $\boldsymbol{A}$, Stacked density plots of saccade latencies of the four observers tested. Markers and error bars show means and SDs for each observer. $\boldsymbol{B}$, Density plot of test offset times relative to saccade onset. We divided the distribution in six presaccadic and three intrasaccadic time windows. The earliest bin collapses all trials in which the test stimulus disappeared $>125$ ms before the saccade; all others are 25 ms wide. C, D, E, Average saccade latency (C), landing site $(\boldsymbol{D})$, and test timing relative to saccade onset $(\boldsymbol{E})$, plotted as a function of cue-test interval and test contrast. Error bars are SEMs.

uncertainty about test stimulus presentation. As a consequence, we could compose a neutral baseline with the same distribution of test stimuli-both in terms of test contrasts and cue-test intervals - as in the saccade condition for each saccade-locked time window (see Materials and Methods, above). Using this procedure, and by locking our analyses of perceptual changes in the saccade condition to the onset of the movement, we isolate the changes in perceptual measures that are due to internal processes evolving with time relative to the onset of the eye movement (i.e., cognitive, sensory, and motor factors related to saccade preparation). The neutral condition also served as an explicit check of how well observers could localize the test patch and, thus, of spatial uncertainty, as they implicitly reported stimulus location by using the corresponding hand (in the saccade condition, the cue indicated the stimulus location). Localization was correct in $99.1 \pm 0.3 \%$ of all neutral trials, and we excluded the few error trials from further analyses to minimize any effects of spatial uncertainty.

We first analyzed how observers' performance in the orientation discrimination task changed with time relative to the eye movement. For each observer and each saccade-locked time window (Fig. 2B), we transformed the orientation reports into a visual sensitivity index, $d^{\prime}$ and averaged it across observers. Starting $\sim 100 \mathrm{~ms}$ before the eye movement, performance increased sharply and beyond the neutral baseline (Fig. $3 A$ ), replicating earlier findings using similar tasks (Castet et al., 2006; Montagnini and Castet, 2007; Rolfs et al., 2011). During the preparation of a saccade, observers became more sensitive to fine orientation differences at the target location.

Having established progressive presaccadic enhancement in visual performance, we now report the dynamics of perceived contrast, the other perceptual report observers made on every trial. In each presaccadic time window, and for each test contrast, we determined the proportion of trials in which observers reported the test stimulus (presented in the presaccadic interval) to have higher contrast than the standard stimulus (presented during fixation). Low test contrasts resulted in a low proportion of "higher" responses; high test contrasts resulted in a high proportion of "higher" responses (brightness-coded in Fig. 3B). In be- tween these two extremes, there was a gradual change in the proportion of responses that we modeled with cumulative Gaussian psychometric functions. The contrast level at which this function has a proportion of 0.5 "higher" reports is the PSE, a sensitive indicator of the test contrast that the observer perceives to be identical to the standard contrast (Carrasco et al., 2004; Liu et al., 2009). We determined the PSE for each presaccadic time window (Fig. 3B, white symbols) and averaged them across observers (Fig. 3C). Long before the saccade, the average PSE was at $23.4 \%$, slightly higher than the standard contrast $(22.4 \%)$. As the saccade approached, however, the average PSE fell continuously, down to $18.0 \%$ when test stimuli appeared around the time of movement onset. A comparison to the neutral baseline data, derived to match the test stimulus parameters prevalent in each saccade-locked time bin (see Materials and Methods, above), reveals significant departures of the PSE starting $100 \mathrm{~ms}$ before the eyes take off (Fig. 3C, bottom; Fig. 3B, colored areas show differences between saccade and neutral condition for each observer). These results suggest that the closer observers are to making a saccade, the higher they perceive the stimulus contrast to be at the target location.

\section{Dynamics of perceptual reports are incompatible with response biases}

We needed to rule out an alternative interpretation of the PSE differences between saccade trials and the neutral baseline-one that is not based on perception, but on changes at the decision stage. In particular, observers might have a bias to report higher contrast when stimuli appear at the target of a saccade. Such a bias would be consistent with a lower PSE in the saccade condition than the neutral control. Explaining the rapid evolution of this difference in the presaccadic interval would be difficult, however, as any putative bias would have to develop rapidly and be timelocked to the saccade, several hundred milliseconds before the observer makes the report. Moreover, although it is possible that the decision about stimulus contrast was time-locked to the saccade (e.g., made right after the eyes land), there is no reason for a putative decisional bias to vary as a function of presaccadic or postsaccadic time; the PSE, however, did change markedly as a function of time relative to the saccade.

In any case, despite its unlikelihood, we assessed the possibility of an evolving bias by analyzing responses to test stimuli that overlapped with saccades, which smear the stationary visual scene across the rapidly moving retina, reducing stimulus visibility. This provides an additional critical test as to whether observers based their reports on what they perceived, rather than on a bias increasing with time, as stimuli presented during the saccade should result in a reduction in perceived contrast, whereas a bias account would predict the opposite. For reports on test stimuli presented (largely or fully) during the saccade, we found that discrimination performance dropped dramatically below the neutral baseline (Fig. 3A, intrasaccadic data), while PSEs increased (a decrease in perceived contrast) and became indistinguishable from both the standard contrast and PSEs in the corresponding neutral 
A

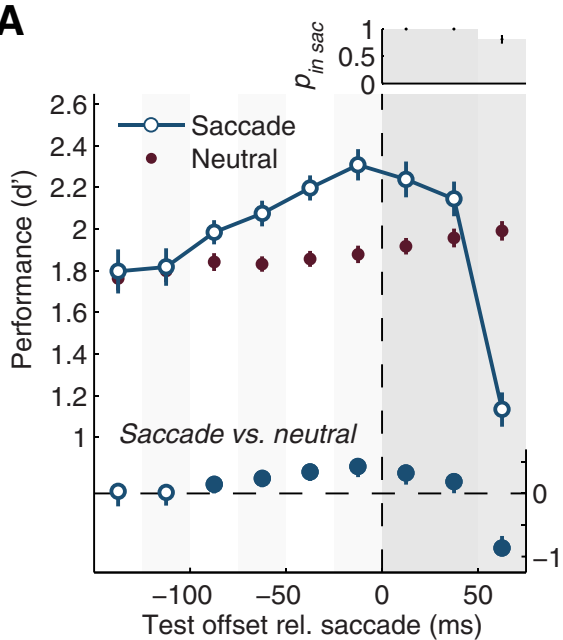

C

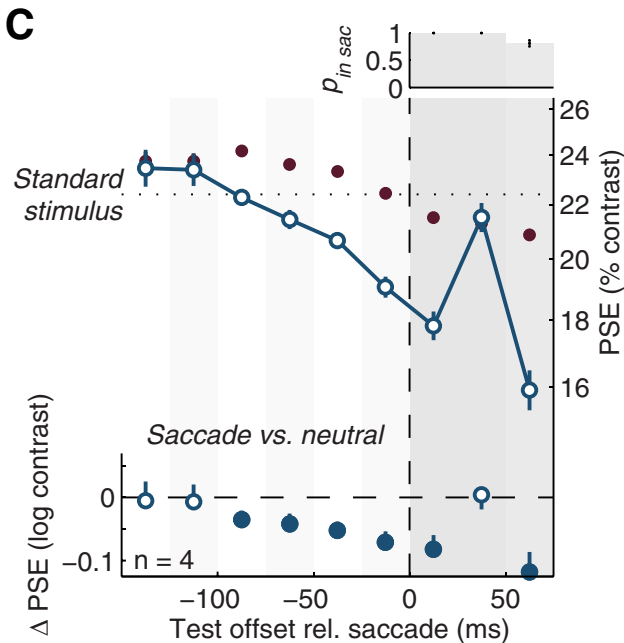

B

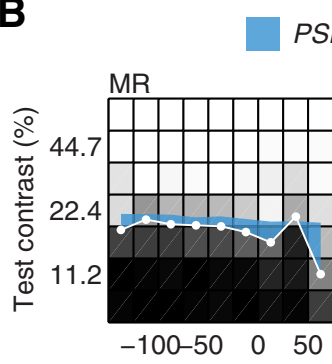

$P S E_{\text {Saccade }}<P S E_{\text {Neutral }}$ AW

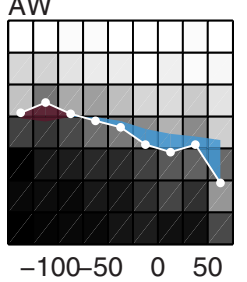

Test offset rel.

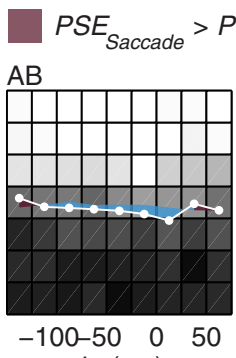

$-100-50 \quad 0 \quad 50$

saccade (ms)

Figure 3. Experiment 1: dynamic perceptual changes before and during saccades. $A$, Average orientation discrimination performance, indexed by $d^{\prime}$, as a function of time relative to saccade onset (time windows defined in Fig. 2B). The neutral baseline was derived for every time window (see Materials and Methods) to control for perceptual changes occurring as a function of test contrast and time after the cue that are not related to the saccade itself. Error bars are SEMs. Bottom, Difference of the saccade condition to the neutral baseline with $95 \% \mathrm{Cls}$. Filled symbols highlight significant differences. The vertical dashed line shows the time of saccade onset; subsequent gray-shaded backgrounds highlight intrasaccadic data. Top, Histogram displays the average likelihood (and SEMs) that the test offset occurred during the saccade (brightness of bars scales with $p_{\text {in sac }}$ ). Stimuli with offsets 50 ms into the saccade completely overlapped with the saccade; stimuli with offsets at earlier times had their onset while the eyes still fixated. Note that the test stimulus orientation was shown during the first $30 \mathrm{~ms}$ of test stimulus only, causing stimuli presented later during the saccade to show a larger decrease in performance. Note also that at least two observers in Experiment 1 had a strong sense of looking at a high-contrast stimulus when they got a peek at the stimulus in the fovea, i.e., when the stimulus duration outlived the saccade (last time bin). $\boldsymbol{B}$, Individual contrast reports and PSEs. For each observer we show the proportion of trials in which observers perceived the test stimulus to have higher contrast than the standard stimulus (22.4\%) as a function of test contrast and test time, coded in shades of gray. White data points show the presaccadic and intrasaccadic PSEs estimated from psychometric functions fitted to these data in each time window. Colored shaded areas highlight the difference of these PSEs to those determined in the matched neutral baseline. This difference generally increases before saccade onset. C, Average perceived contrast, expressed as PSE, as a function of time relative to saccade onset. Conventions as in panel $\boldsymbol{A}$.

baseline (Fig. 3C, intrasaccadic data). These results show that we measured continuous perceptual changes as an observer prepares and executes a saccadic eye movement, not a bias to favor one response ("higher") over another ("lower") in the comparison of test and standard.

\section{Presaccadic perceptual changes occur earlier than during covert attention}

The observed enhancement of visual performance and perceived contrast before saccades have a similar magnitude as the corresponding effects in studies of involuntary (exogenous, transient; Carrasco et al., 2004) and voluntary (endogenous, sustained; Liu et al., 2009) covert attention. Spatial cueing of involuntary attention affects perception $80-120 \mathrm{~ms}$ after the cue and only if the cue is presented peripherally, adjacent to the stimulus; spatial cueing of voluntary covert attention affects perception only $300 \mathrm{~ms}$ after the cue (Müller and Rabbitt, 1989; Nakayama and Mackeben, 1989; Cheal and Lyon, 1991a,b; Hopfinger and West, 2006; Ling and Carrasco, 2006; Liu et al., 2007; Busse et al., 2008; for review, see Carrasco, 2011). In our task, however, the average saccade latency was $\sim 190 \mathrm{~ms}$ and perceptual enhancement preceded saccades by $\sim 100$ $\mathrm{ms}$. In fact, when we restricted our analyses to test stimuli presented
$60 \mathrm{~ms}$ after the central cue onset, we still found robust changes in performance and perceived contrast, time-locked to the saccade (Fig. 4). Based on the covert attention literature, one would not expect any impact of either involuntary or voluntary covert attention on either performance or appearance this shortly after a central spatial cue.

To confirm that these temporal dynamics were indeed faster than expected from a voluntary deployment of covert attention, we investigated whether saccade preparation was necessary to obtain the described perceptual changes, or alternatively, if they were driven by the observers' knowledge of the test location, which was given by the spatial cue. In Experiment 2, therefore, we asked observers to maintain fixation while covertly attending to the indicated target location, rather than making a saccade in response to the spatial cue. In addition to equating temporal uncertainty about the test stimulus onset, this covert attention condition also equated spatial uncertainty with that of the saccade condition in Experiment 1; as in Experiment 1, spatial uncertainty was negligible (99.3 \pm $0.5 \%$ correct localization). The rationale of this experiment was the following: if the cue-based knowledge of the spatial location of the test stimulus underlied the results in Experiment 1, then for both discrimination performance and perceived contrast the 
A

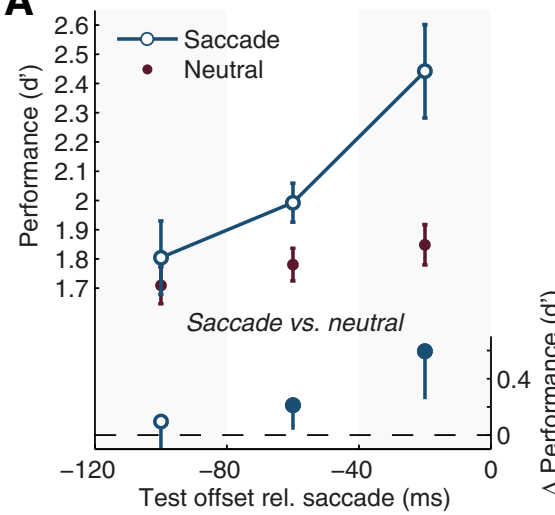

B

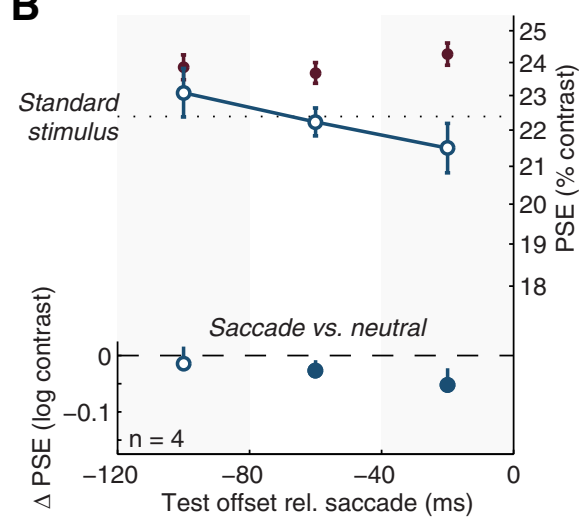

Figure 4. Rapid perceptual changes during saccade preparation. This reanalysis of data from Experiment 1 is restricted to test stimuli presented $60 \mathrm{~ms}$ after cue onset. $\boldsymbol{A}, \boldsymbol{B}$, Changes in orientation discrimination performance measured as $d^{\prime}(\boldsymbol{A})$ and perceived contrast measured in terms of PSE $(\boldsymbol{B})$ are shown as a function of time before the saccade. Conventions as in Figure 2. By definition, this analysis excludes any potential contributions of time relative to the cue to the observed effects. We chose the $60 \mathrm{~ms}$ cue-test interval to be able to examine perceptual changes very early after the cue, while having sufficient data to allow for an analysis of performance and perceived contrast in several equidistant time windows before the saccade. Note that the reduction in temporal resolution (from 25 to $40 \mathrm{~ms}$ ) could wash out any potential effects. Nevertheless, substantial differences between the saccade condition and the neutral baseline develop gradually and become significant as time approaches the saccade.

in time (Fig. $5 A$, bottom), and the only differences in PSEs (Fig. 5B, bottom) were in the opposite direction and occurred in different time windows than the difference between the saccade condition and its neutral baseline.

To ensure that observers were deploying covert attention in this task, we retested the same observers with a broader range of cue-test intervals while they maintained fixation. We did obtain reliable PSE differences between the neutral and covert attention condition if cue and test were separated by $500 \mathrm{~ms}$ ( $\triangle$ PSEs were $-0.008 \pm 0.019,-0.023 \pm 0.023$, and $-0.061 \pm 0.030$ units $\log$ contrast, for 100,300 , and $500 \mathrm{~ms}$ cue-test intervals, respectively). That is, provided sufficient time to allocate voluntary covert attention, observers did perceive test stimuli to be of higher contrast than in a neutral condition, in which the target location was not precued. This finding is consistent with a previous study (Liu et al.,

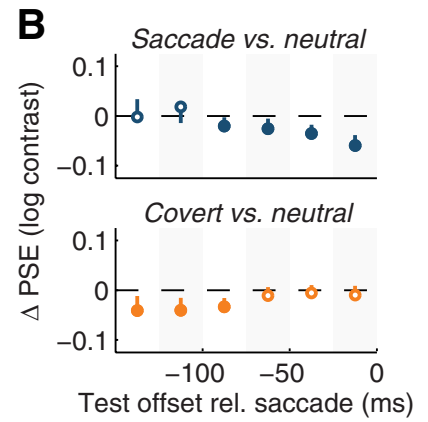

Figure 5. Experiment 2: spatial cueing does not account for the rapid evolution of performance and appearance before saccades. $\boldsymbol{A}, \boldsymbol{B}$, Top, Difference in orientation discrimination performance $(\boldsymbol{A})$ and PSEs between saccade and derived neutral baselines $(\boldsymbol{B})$ in Experiment 1 (Fig. 2), replotted for the three observers who also participated in Experiment 2. Bottom, Difference between the covert attention condition and its neutral baseline, computed separately for each presaccadic time window, analogously to the neutral baseline in Experiment 1. These data, thus, display the differences between neutral and covert conditions expected based on the cue-test timings and test contrasts prevalent in each time window of the saccade condition in Experiment 1. Conventions as in Figure 2, bottom.

magnitude of the difference between this covert attention condition and the neutral baseline should be equal to that between the saccade condition and the neutral baseline obtained in Experiment 1.

The data clearly show that this was not the case. We first replotted data from Experiment 1 for the three observers who also participated in this covert attention experiment. We confirmed reliable differences in performance and perceived contrast, increasing as the saccade nears (Fig. 5, top). Next, we computed presaccadic baselines from the data in the covert attention experiment, using the same procedure as for the neutral data in all other experiments, i.e., by weighting each combination of test contrast and cue-test interval in the covert and neutral conditions according to the prevalence of these combinations of test contrast and cue-test interval in a particular time bin of the saccade condition (see Materials and Methods, above). Then, we averaged the difference between the covert attention condition and its neutral baseline. There were no significant differences in performance between covert and neutral conditions at any point
2009), and is in agreement with the literature on the time course of voluntary covert attention (Müller and Rabbitt, 1989; Nakayama and Mackeben, 1989; Ling and Carrasco, 2006; Liu et al., 2007). Hence, these results show that the rapid perceptual changes evident before saccades (Experiments 1 and 3) observed for test stimuli presented as early as $60 \mathrm{~ms}$ after the cue cannot be accounted for by spatial cueing of the test location - the speed of perceptual changes before saccades far exceeds that of voluntary covert attention. Saccade preparation is responsible for the rapid evolution of performance and perceived contrast.

\section{Presaccadic appearance changes for contrast judgments alone and without a mask}

Experiment 3 addressed two possible concerns with Experiment 1. First, we ruled out that observers based their contrast report on their performance in the discrimination task, reporting a highcontrast test stimulus when they were confident about the orientation of the stimulus, a potential account of why discrimination performance and perceived contrast covaried in the first experiment. Second, we eliminated any possible effect of the mask on the contrast judgment. Two previous studies have shown that a mask that effectively renders stimuli invisible during fixation may become less effective when presented within a tenth of a second before a saccade (De Pisapia et al., 2010; Hunt and Cavanagh, 2011). There was a possibility, therefore, that the test Gabor in our study appeared to have a higher contrast because the mask could have lost its impact. We repeated Experiment 1 with two critical changes: First, the observers only performed one perceptual task: a contrast judgment. Second, both standard and test stimuli consisted only of a Gabor presented for $50 \mathrm{~ms}$. That is, we no longer used the subsequent orientation-noise mask initially introduced to avoid ceiling performance in the orientation discrimination task.

The results of Experiment 3 show that neither the concurrent discrimination task nor the presence of a mask could account for the observed change in the PSE before a saccade. Both average saccade latencies (190 \pm 7 ms; Fig. 6A) and amplitudes (9.0 \pm $0.2^{\circ}$ ) were remarkably similar to those observed in Experiment 1 and, as before, varied with test contrast and onset time (data not 
A

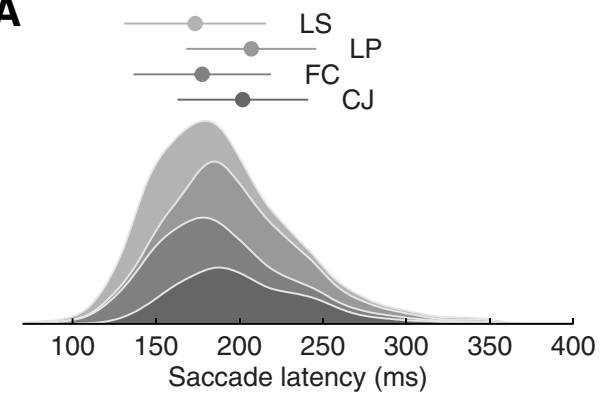

B

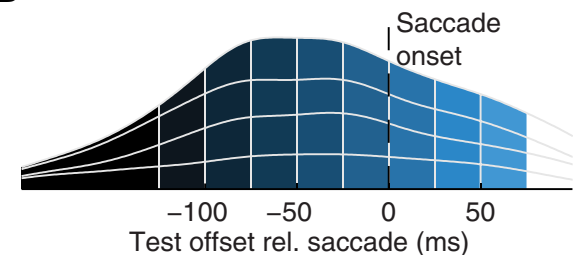

D

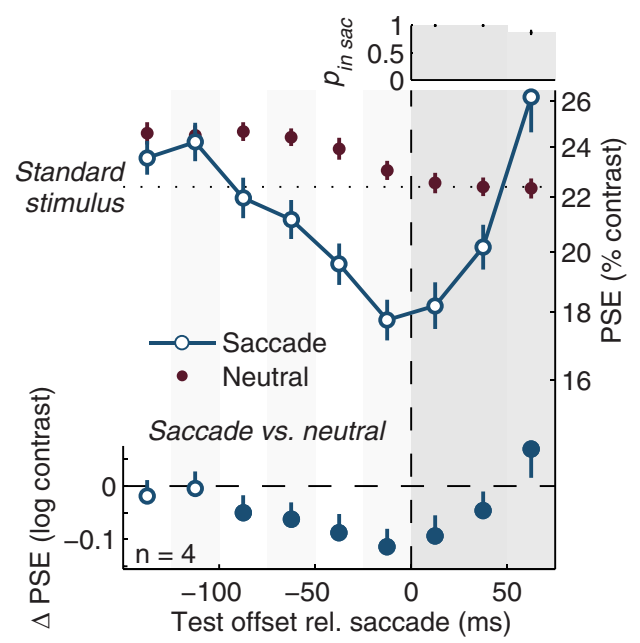

C

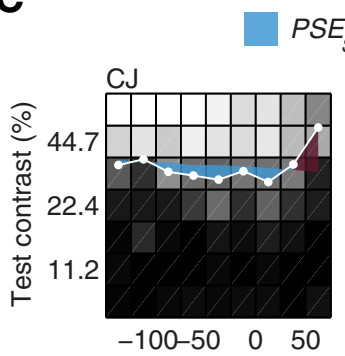

$P S E_{\text {Saccade }}<P S E_{\text {Neutral }}$

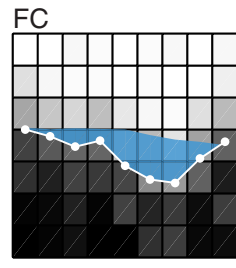

$-100-50 \quad 0 \quad 50$

Test offset rel. saccade (ms)

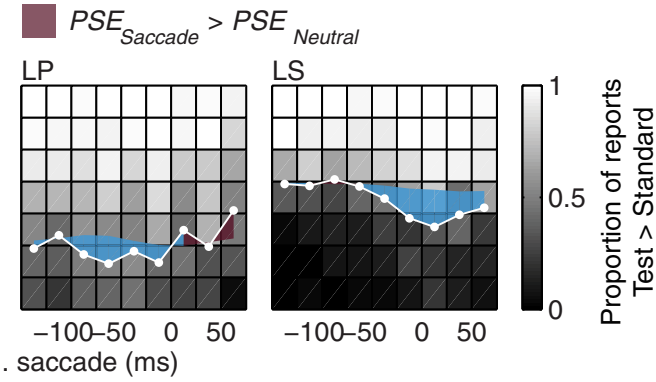

Figure 6. Experiment 3: testing the changes in perceived contrast before saccades in the absence of a discrimination task and without a mask. $A$, Stacked density plots of saccade latencies of the four observers tested. Markers and error bars show each observer's mean and SD. B, Density plot of test offset times relative to saccade onset, divided into six presaccadic and three intrasaccadic time windows. $\boldsymbol{C}$, Individual contrast reports and PSEs. D, Average perceived contrast as a function of time relative to saccade onset. All conventions as in Figure 3.

shown). Test offset times distributed broadly over the presaccadic interval (52.6\% of the trials; Fig. $6 B$ ), allowing us to use the same temporal binning of presaccadic and intrasaccadic test times as in Experiment 1. Stimulus localization was correct in $98.3 \pm 1.2 \%$ of all neutral trials and we excluded trials with errors to minimize any contribution of spatial uncertainty. The high degree of similarity of these results to those in the Experiment 1 suggests that observers in the two experiments pursued similar strategies performing the task.

We determined PSEs for each presaccadic time window along with a baseline derived from neutral trials that we constructed for each of these bins (Fig. 6C). We then averaged PSEs across observers (Fig. 6D). Long before the saccade, the average PSE was $23.6 \%$, slightly higher than the standard contrast (22.4\%) and not different from its neutral baseline. With time approaching the onset of the eye movement, the average PSE fell markedly, down to $17.8 \%$. Whereas the difference to the neutral baseline was insignificant $>100 \mathrm{~ms}$ before the saccade, we observed increasing and reliable differences during the last $100 \mathrm{~ms}$ before the saccade (Fig. 6D, bottom). As in Experiment 1, when test stimuli overlapped with the saccade in time, PSEs increased and became indistinguishable from both the neutral baseline and the standard contrast (intrasaccadic data in Fig. 6D), validating that observers based their reports on perception rather than on response biases. Having excluded unlikely but potential confounds by a concurrent discrimination task or the presence of a mask, this experiment strengthens our conclusion that observers perceive the same physical stimulus as having higher contrast when it appears at the saccade target shortly before a saccade.

\section{Discussion}

Implementing a novel behavioral paradigm, inspired by studies of phenomenological correlates of covert attention (Carrasco et al., 2004; Liu et al., 2009), we observed rapid presaccadic changes in both orientation discrimination performance and the subjective perception of stimulus contrast. Just before the eyes move, observers become more sensitive to the orientation of a stimulus presented at the saccade target, and perceive this stimulus to have a higher contrast—standing out more from the backgroundthan when they see that same stimulus during a fixation period (Experiments 1 and 3) or when the target location is cued but no saccade is made and there is no time to deploy covert attention (Experiment 2). The changes in visual sensitivity and perceived contrast have similar temporal dynamics, unfolding around a tenth of a second before the eyes start moving.

We have ruled out alternative interpretations of these data. First, the results cannot be explained by a bias to report a cued stimulus more often as having higher contrast than a stimulus preceded by a neutral cue. Any such decision-based account fails to explain: (1) the continuous changes in perceptual reports before the saccade (Figs. $3 A, C ; 6 D)$, (2) the drastic reversal in contrast reports when test stimuli temporally overlapped with the saccade (Fig. $3 A, C ; 6 D$ ), and (3) the absence of an increase in PSE when the test location was cued but no saccade was executed (Fig. 5). Second, changes in perceived contrast were not the result of presaccadic changes in the effectiveness of a mask stimulus (De Pisapia et al., 2010; Hunt and Cavanagh, 2011) used in Experiment 1 to limit discrimination per- 
formance. We obtained the same results in the absence of a mask (Experiment 2). Finally, we obtained identical results when observers made contrast judgments only (Experiment 2), ruling out biases to report higher perceived contrast when performance is high in the orientation discrimination task (Carrasco et al., 2004).

Our results, therefore, provide the first direct behavioral evidence showing that saccade preparation (or a corollary signal) affects subjective experience of visual intensity-it increases perceived stimulus strength in a manner akin to an increase in stimulus contrast. We put forward a linking hypothesis relating presaccadic changes in neural responses not only to changes in visual performance but also to the subjective appearance of contrast. Preparing a saccade to a visual stimulus increases the strength of its neural representation in many visual brain areas (Boch and Fischer, 1983; Chelazzi et al., 1993; Moore et al., 1998; Sheinberg and Logothetis, 2001; Mazer and Gallant, 2003; Supèr et al., 2004), and these neural changes closely mimic those in response to an increase in physical stimulus salience ( $\mathrm{Li}$ and Basso, 2008). By measuring perceived contrast, we revealed correlates of these neural changes in observers' perceptual reports. Movement preparation thus, like covert attention (Treue, 2004; Carrasco, 2009, 2011), alters sensory processing to create a perceptual experience that integrates visual signals and behavioral priority.

What is the neural signature that gives rise to an increase in perceived contrast? The most parsimonious account of the present results appears to be a common underlying mechanism driving the dynamics of both visual performance and stimulus appearance. Assuming that the increase in stimulus discriminability results from enhanced visual sensitivity, i.e., a higher signal-to-noise ratio at an early stage of visual processing, enhanced perceived contrast could be a direct consequence. A direct coupling between performance and appearance is not trivial, however, and reliable effects of attentional manipulation on visual performance without a concurrent change in the subjective appearance of a stimulus have been reported for color hue (Fuller and Carrasco, 2006). Conceivable alternatives for the neural basis of changes in perceived contrast include the response magnitude of populations of visual neurons processing the stimulus or the coherence of neural responses across areas. In a combined effort, behavioral, physiological, and computational studies may test the validity of these plausible alternative accounts.

The immediacy of perceptual changes when observers prepare a saccade stresses the pronounced impact of action on visual perception. Previous studies assessed visual sensitivity and appearance during the involuntary (Carrasco et al., 2004; Gobell and Carrasco, 2005; Fuller and Carrasco, 2006; Liu et al., 2006) or voluntary deployment of covert attention, when observers monitored peripheral locations without moving their eyes (Liu et al., 2009; Abrams et al., 2010). The studies on voluntary deployment of attention used long cue-test intervals, as it takes $\sim 300 \mathrm{~ms}$ following cue onset to be deployed (Müller and Rabbitt, 1989; Nakayama and Mackeben, 1989; Cheal and Lyon, 1991a,b; Hopfinger and West, 2006; Ling and Carrasco, 2006; Liu et al., 2007; Busse et al., 2008), much longer than the average saccade latency in the present dataset. In fact, we found significant changes in both performance and appearance within $60 \mathrm{~ms}$ after the onset of a symbolic cue, but only when the observer prepared a saccade. Moreover, the transient deployment of involuntary attention cannot be responsible for the observed effects, because we did not use a peripheral cue (which is necessary to elicit transient attention), the timing of our effects was slightly faster than that of involuntary attention (Müller and Rabbitt, 1989; Nakayama and
Mackeben, 1989; Cheal and Lyon, 1991a,b; Hopfinger and West, 2006; Busse et al., 2008) and any stimulus onset was the same for the saccade, the neutral, and the covert attention conditions. Thus, either saccade preparation or the decision to make a saccade boosts visual processing of information at the peripheral target, with a delay much shorter than that of voluntary covert attention.

The difference in temporal dynamics could be interpreted as evidence for a dissociation of covert attention and saccade preparation, which previous behavioral studies have suggested (Hunt and Kingstone, 2003; Belopolsky and Theeuwes, 2009). Indeed, several neurophysiological studies have found that distinct neural populations in the frontal eye fields or the superior colliculus may control covert attention and saccade preparation (Ignashchenkova et al., 2004; Juan et al., 2004; Thompson et al., 2005; Gregoriou et al., 2012), potentially giving rise to differences in their perceptual correlates. Alternatively, despite the salient differences in the time course of perceptual changes during covert voluntary attention and saccade preparation, their neural origins may be common (Rizzolatti et al., 1987). We know that brain areas implementing the generation of saccadic eye movements also play a vital role in the control of covert attention (Moore and Fallah, 2001; Cavanaugh and Wurtz, 2004; Wardak et al., 2004, 2006; Müller et al., 2005; Balan and Gottlieb, 2009; Lovejoy and Krauzlis, 2010). Subthreshold stimulation of a neural population in the frontal eye fields (Moore and Fallah, 2001) or the superior colliculus (Cavanaugh and Wurtz, 2004; Müller et al., 2005) causes reliable performance benefits for stimuli presented at the corresponding retinal location, probably through feedback to visual cortex. When signals in these areas rise to movement threshold during the preparation of a saccade, feedback signals may affect visual processing at peripheral retinal locations with minimal delay (Armstrong and Moore, 2007), similar to peripheral visual cues that rapidly alter visual processing in studies of involuntary attention (for review, see Carrasco, 2011). We speculate that voluntary attention, if controlled by the same areas but by different neuronal subpopulations, may need more time to develop and exert its influence on visual processing when a saccade is not being programmed.

The rapid changes in visual performance and appearance during the preparation of saccades reveal a tight collaboration between sensation and movement. The functional benefits of this tight coupling are indisputable. We move the eyes to inspect potentially relevant information in the visual scene. Enhancing the discriminability and signal strength of stimuli at the target location as soon as the preparation of an eye movement has started facilitates immediate visual analysis and transsaccadic integration of visual information (Hamker et al., 2008; Rolfs et al., 2011). The present results showcase this functional integration of perception and action and urge us to study them in an active-perception framework. The preparation of saccades not only affects our visual performance but, by intensifying the sensory impression of a stimulus, it also alters the way the world appears to us.

\section{References}

Abrams J, Barbot A, Carrasco M (2010) Voluntary attention increases perceived spatial frequency. Atten Percept Psychophys 72:1510-1521. CrossRef Medline

Armstrong KM, Moore T (2007) Rapid enhancement of visual cortical response discriminability by microstimulation of the frontal eye field. Proc Natl Acad Sci U S A 104:9499-9504. CrossRef Medline

Balan PF, Gottlieb J (2009) Functional significance of nonspatial informa- 
tion in monkey lateral intraparietal area. J Neurosci 29:8166-8176. CrossRef Medline

Belopolsky AV, Theeuwes J (2009) When are attention and saccade preparation dissociated? Psychol Sci 20:1340-1347. CrossRef Medline

Boch R, Fischer B (1983) Saccadic reaction times and activation of the prelunate cortex: parallel observations in trained rhesus monkeys. Exp Brain Res 50:201-210. Medline

Brainard DH (1997) The psychophysics toolbox. Spat Vis 10:433-436. CrossRef Medline

Busse L, Katzner S, Treue S (2008) Temporal dynamics of neuronal modulation during exogenous and endogenous shifts of visual attention in macaque area MT. Proc Natl Acad Sci U S A 105:16380-16385. CrossRef Medline

Carrasco M (2009) Cross-modal attention enhances perceived contrast. Proc Natl Acad Sci U S A 106:22039-22040. CrossRef Medline

Carrasco M (2011) Visual attention: the past 25 years. Vision Res 51:14841525. CrossRef Medline

Carrasco M, Penpeci-Talgar C, Eckstein M (2000) Spatial covert attention increases contrast sensitivity across the CSF: support for signal enhancement. Vision Res 40:1203-1215. CrossRef Medline

Carrasco M, Ling S, Read S (2004) Attention alters appearance. Nat Neurosci 7:308-313. CrossRef Medline

Castet E, Jeanjean S, Montagnini A, Laugier D, Masson GS (2006) Dynamics of attentional deployment during saccadic programming. J Vis 6:196212. CrossRef Medline

Cavanaugh J, Wurtz RH (2004) Subcortical modulation of attention counters change blindness. J Neurosci 24:11236-11243. CrossRef Medline

Cheal ML, Lyon DR (1991a) Central and peripheral precuing of forcedchoice discrimination. Q J Exp Psychol A 43:859-880. CrossRef Medline

Cheal ML, Lyon DR (1991b) Importance of precue location in directing attention. Acta Psychologica 76:201-211. CrossRef Medline

Chelazzi L, Miller EK, Duncan J, Desimone R (1993) A neural basis for visual search in inferior temporal cortex. Nature 363:345-347. CrossRef Medline

Cornelissen FW, Peters EM, Palmer J (2002) The Eyelink Toolbox: eye tracking with MATLAB and the Psychophysics Toolbox. Behav Res Methods Instrum Comput 34:613-617. CrossRef Medline

De Pisapia N, Kaunitz L, Melcher D (2010) Backward masking and unmasking across saccadic eye movements. Curr Biol 20:613-617. CrossRef Medline

Deubel H (2008) The time course of presaccadic attention shifts. Psychological Res 72:630-640. CrossRef Medline

Deubel H, Schneider WX (1996) Saccade target selection and object recognition: evidence for a common attentional mechanism. Vision Res 36: 1827-1837. CrossRef Medline

Engbert R, Mergenthaler K (2006) Microsaccades are triggered by low retinal image slip. Proc Natl Acad Sci U S A 103:7192-7197. CrossRef Medline

Fuller S, Carrasco M (2006) Exogenous attention and color perception: performance and appearance of saturation and hue. Vision Res 46:40324047. CrossRef Medline

Gobell J, Carrasco M (2005) Attention alters the appearance of spatial frequency and gap size. Psychol Sci 16:644-651. CrossRef Medline

Gregoriou GG, Gotts SJ, Desimone R (2012) Cell-type-specific synchronization of neural activity in FEF with V4 during attention. Neuron 73:581594. CrossRef Medline

Hamker FH (2005) The reentry hypothesis: the putative interaction of the frontal eye field, ventrolateral prefrontal cortex, and areas V4, IT for attention and eye movement. Cereb Cortex 15:431-447. CrossRef Medline

Hamker FH, Zirnsak M, Calow D, Lappe M (2008) The peri-saccadic perception of objects and space. PLoS Comput Biol 4:e31. CrossRef Medline

Herrmann K, Montaser-Kouhsari L, Carrasco M, Heeger DJ (2010) When size matters: attention affects performance by contrast or response gain. Nat Neurosci 13:1554-1559. CrossRef Medline

Hopfinger JB, West VM (2006) Interactions between endogenous and exogenous attention on cortical visual processing. Neuroimage 31:774-789. CrossRef Medline

Hunt AR, Cavanagh P (2011) Remapped visual masking. J Vis 11:13. CrossRef Medline

Hunt AR, Kingstone A (2003) Covert and overt voluntary attention: linked or independent? Brain Res Cogn Brain Res 18:102-105. CrossRef Medline
Ignashchenkova A, Dicke PW, Haarmeier T, Thier P (2004) Neuronspecific contribution of the superior colliculus to overt and covert shifts of attention. Nat Neurosci 7:56-64. CrossRef Medline

Juan CH, Shorter-Jacobi SM, Schall JD (2004) Dissociation of spatial attention and saccade preparation. Proc Natl Acad Sci U S A 101:15541-15544. CrossRef Medline

Kowler E, Anderson E, Dosher B, Blaser E (1995) The role of attention in the programming of saccades. Vision Res 35:1897-1916. CrossRef Medline

Li X, Basso MA (2008) Preparing to move increases the sensitivity of superior colliculus neurons. J Neurosci 28:4561-4577. CrossRef Medline

Ling S, Carrasco M (2006) When sustained attention impairs perception. Nat Neurosci 9:1243-1245. CrossRef Medline

Liu T, Fuller S, Carrasco M (2006) Attention alters the appearance of motion coherence. Psychon Bull Rev 13:1091-1096. CrossRef Medline

Liu T, Stevens ST, Carrasco M (2007) Comparing the time course and efficacy of spatial and feature-based attention. Vision Res 47:108-113. CrossRef Medline

Liu T, Abrams J, Carrasco M (2009) Voluntary attention enhances contrast appearance. Psychol Sci 20:354-362. CrossRef Medline

Lovejoy LP, Krauzlis RJ (2010) Inactivation of primate superior colliculus impairs covert selection of signals for perceptual judgments. Nat Neurosci 13:261-266. CrossRef Medline

Luck SJ (2004) Understanding awareness: one step closer. Nat Neurosci 7:208-209. CrossRef Medline

Mazer JA, Gallant JL (2003) Goal-related activity in V4 during free viewing visual search: evidence for a ventral stream visual salience map. Neuron 40:1241-1250. CrossRef Medline

Montagnini A, Castet E (2007) Spatiotemporal dynamics of visual attention during saccade preparation: independence and coupling between attention and movement planning. J Vis 7:8.1-16. CrossRef Medline

Moore T, Fallah M (2001) Control of eye movements and spatial attention. Proc Natl Acad Sci U S A 98:1273-1276. CrossRef Medline

Moore T, Tolias AS, Schiller PH (1998) Visual representations during saccadic eye movements. Proc Natl Acad Sci U S A 95:8981-8984. CrossRef Medline

Müller HJ, Rabbitt PM (1989) Reflexive and voluntary orienting of visual attention: time course of activation and resistance to interruption. J Exp Psychol Hum Percept Perform 15:315-330. CrossRef Medline

Müller JR, Philiastides MG, Newsome WT (2005) Microstimulation of the superior colliculus focuses attention without moving the eyes. Proc Natl Acad Sci U S A 102:524-529. CrossRef Medline

Nakayama K, Mackeben M (1989) Sustained and transient components of focal visual attention. Vision Res 29:1631-1647. CrossRef Medline

Pelli DG (1997) The VideoToolbox software for visual psychophysics: transforming numbers into movies. Spat Vis 10:437-442. CrossRef Medline

Pestilli F, Ling S, Carrasco M (2009) A population-coding model of attention's influence on contrast response: estimating neural effects from psychophysical data. Vision Res 49:1144-1153. CrossRef Medline

Rizzolatti G, Riggio L, Dascola I, Umiltá C (1987) Reorienting attention across the horizontal and vertical meridians: evidence in favor of a premotor theory of attention. Neuropsychologia 25:31-40. CrossRef Medline

Rolfs M, Jonikaitis D, Deubel H, Cavanagh P (2011) Predictive remapping of attention across eye movements. Nat Neurosci 14:252-256. CrossRef Medline

Sheinberg DL, Logothetis NK (2001) Noticing familiar objects in real world scenes: the role of temporal cortical neurons in natural vision. J Neurosci 21:1340-1350. Medline

Supèr H, van der Togt C, Spekreijse H, Lamme VA (2004) Correspondence of presaccadic activity in the monkey primary visual cortex with saccadic eye movements. Proc Natl Acad Sci U S A 101:3230-3235. CrossRef Medline

Thompson KG, Biscoe KL, Sato TR (2005) Neuronal basis of covert spatial attention in the frontal eye field. J Neurosci 25:9479-9487. CrossRef Medline

Treue S (2004) Perceptual enhancement of contrast by attention. Trends Cogn Sci 8:435-437. CrossRef Medline

Wardak C, Olivier E, Duhamel JR (2004) A deficit in covert attention after parietal cortex inactivation in the monkey. Neuron 42:501-508. CrossRef Medline

Wardak C, Ibos G, Duhamel JR, Olivier E (2006) Contribution of the mon- 
key frontal eye field to covert visual attention. J Neurosci 26:4228-4235. CrossRef Medline

Watson AB, Pelli DG (1983) QUEST: a Bayesian adaptive psychometric method. Perception and Psychophysics 33:113-120. CrossRef Medline

Wichmann FA, Hill NJ (2001a) The psychometric function. I. Fitting, sam- pling, and goodness of fit. Percept Psychophys 63:1293-1313. CrossRef Medline

Wichmann FA, Hill NJ (2001b) The psychometric function. II. Bootstrapbased confidence intervals and sampling. Percept Psychophys 63:13141329. CrossRef Medline 